ISSN: 2600-5859

\title{
Proceso enseñanza de la técnica de carrera en atletas de la categoría 10 a 11 años.
}

\begin{abstract}
(c) (i) (2)
BY NC SA
\end{abstract}

Proceso de enseñanza de la técnica de carrera en atletas de la categoría 10 a 11 años.

Chiluisa Lagla Diego Mauricio. ${ }^{1}$, Castro Pantoja Edison Andrés. ${ }^{2}$, Paz Puente Fabricio

Alexander. ${ }^{3} \&$ Barrera Cueva Janeth del Carmen. ${ }^{4}$

Recibido:05-01-2020 / Revisado: 02-02-2020 /Aceptado: 20-02-2020/ Publicado: 06-03-2020

\begin{abstract}
.
DOI: $\underline{\text { https://doi.org/10.33262/concienciadigital.v3i1.2.1191 }}$

With the aim of establishing adaptations in the career technique teaching process that allows a correct performance of the flat racing technique, this research work was carried out, That it is focused on the process that lead to the learning of the career technique, where a prospective analysis was carried out, of a qualitative-quantitative nature that allowed us to inquire about the phenomenon studied. A critical, reflective and purposeful investigation was carried out about the teaching methods and methodological procedures, aimed at a significant learning of the technique of flat runs, these fundamental elements for the application of observation during technical performance. The results of the investigation verified the existing deficiencies in the process. For the validity of the evaluated exercises of the observation sheet, they were checked through the statistical processes of the Student Test and the corresponding analysis and interpretation, this led us to the execution of parameters that helped the development and improvement of the technical component, creating thus an exercise guide.
\end{abstract}

Keywords: Observation sheet, athletics, teaching process, running technique

\section{Resumen.}

Con el objetivo de establecer adecuaciones en el proceso de enseñanza de la técnica de carrera que permita un correcto desempeño de la técnica de las carreras planas se realizó el presente trabajo

${ }^{1}$ Universidad Técnica de Ambato. Carrera Cultura Física. Ambato, Ecuador, dcmauricio2405@gmail.com

${ }^{2}$ Universidad Politécnica Estatal del Carchi. Centro de Cultura Física. Carchi, Ecuador, titoruner@htomail.com

${ }^{3}$ U.E Isaac Acosta. Carchi, Ecuador, g_unitpazf@hotmail.com

${ }^{4}$ Universidad Técnica de Ambato. Carrera Cultura Física. Ambato, Ecuador. janethbarrerac@ @otmail.com 
investigativo, Que está centrado en el proceso que conllevan al aprendizaje de la técnica de carrera, donde se llevó a cabo un análisis prospectivo, de naturaleza cualitativa-cuantitativa que permitió indagar acerca del fenómeno estudiado. Se ejecutó una investigación crítica, reflexiva y propositiva acerca de las formas de enseñanza y procedimientos metodológicos, encaminados a un aprendizaje significativo de la técnica de las carreras planas, elementos estos fundamentales para la aplicación de la observación durante el desempeño técnico. Los resultados de la investigación comprobaron las deficiencias existentes en el proceso. Para la validez de los ejercicios evaluados de la ficha de observación se comprobaron a través de los procesamientos estadísticos de Test de Student y el concerniente análisis e interpretación, esto nos condujo a la ejecución de parámetros que ayudaron al desarrollo y mejoría del componente técnico, creando de este modo una guía de ejercicios.

Palabras claves: Ficha de observación, Atletismo, Proceso de enseñanza, Técnica de carrera

\section{Antecedentes.}

La enseñanza del atletismo está dirigida hacia cuatro áreas fundamentales que agrupan pruebas características por su ejecución, dentro de estas podemos encontrar la caminata o marcha deportiva, las carreras, los saltos y los lanzamientos. En todas estas pruebas se imparten una variada gama de contenidos facilitando profundizar a los estudiantes en los conocimientos de este deporte. Teniendo en cuenta la rapidez con que se desarrolla el contexto actual y la exigua preparación teórica que muestran los profesores noveles durante el PEA se constata la necesidad de seguir profundizando en la enseñanza de los fundamentos técnicos que sustentan el desarrollo del atletismo en su contexto actual (Montoro Bombú, Quizhpe Luzuriaga, et al., 2018 citado en Lagla Chiluisa 2020).

Por otra parte, en los últimos años se ha podido observar un aumento en el número de sujetos que practican diferentes modalidades del atletismo recreativo con una base aeróbica predominante, así como la organización de diferentes eventos deportivos para la demostración de la técnica (Alex Mauricio Carrillo Aguagallo et al., 2018 citado en Lagla Chiluisa 2020).

Para que todo este proceso pueda realizarse tiene que existir la permanencia del deportista en el proceso que participa, es un componente indispensable en el logro de altos rendimientos deportivos y es parte indispensable de la preparación.(Inelvis Romero Pileta et al., 2019 citado en Lagla Chiluisa 2020). La investigación se cuenta con diferentes antecedentes relacionados con el objeto de estudio. En tal sentido mediante el método de revisión de fuentes documentales se pudo constatar la tesis de (Cevallos Camacho, 2014 citado en Lagla Chiluisa 2020). en su tema de investigación:

Programa de iniciación deportiva en el atletismo para mejorar los procesos de enseñanza aprendizaje en los alumnos de la unidad educativa Machachi, cantón Mejía, provincia de Pichincha, durante el año lectivo 2014- 2015, en su investigación el autor realiza búsquedas acerca de las metodologías para la enseñanza en la iniciación al atletismo, a su vez concluye que: las actividades didácticas ludo técnicas son herramientas valiosas que permiten guiar adecuadamente el proceso de enseñanza aprendizaje de iniciación al atletismo de medio fondo, para obtener nuevos talentos deportivos. 
El trabajo investigativo otorga beneficios tanto a los deportistas como entrenadores, debido a que se da a conocer un método innovador y adecuado de entrenamiento para mejorar los fundamentos técnicos.

Otro de los textos consultados fue la investigación: Guía metodológica para el perfeccionamiento de la técnica de la carrera en atletas fondistas del Club Trotahacheros del Municipio de Tuquerres Nariño, dan a conocer que una guía metodológica para el perfeccionamiento en la técnica de carrera en atletas permite mejorar los procesos de formación deportiva, puesto que a partir del conocimiento científico combinado con los saberes empíricos se pretende alcanzar un mejor rendimiento deportivo. Además, deducen que "la ejecución correcta de la técnica de la carrera contribuye significativamente al resultado deportivo (Tobar, M. A., \& Zurata, 2013 citado en Lagla Chiluisa 2020).

En su tema de investigación: La evaluación pedagógica y su incidencia en el desarrollo competitivo en las pruebas de medio fondo en los atletas pre-juveniles de la Federación Deportiva de Loja periodo 2013; señala que al hablar de evaluación pedagógica se refiere a un medio que sirve para comprobar el proceso de enseñanza - aprendizaje, por lo que toma en cuenta el tipo de evaluación a aplicar de acuerdo al grado de eficiencia en el desarrollo competitivo en las pruebas de medio fondo para así evitar el fracaso en el desenvolvimiento competitivo del atleta (Narváez Galván 2013 citado en Lagla Chiluisa 2020).

Además, da a conocer que los controles pedagógicos si tienen una importancia significativa dentro de la macro planificación realizada por los entrenadores del medio. Junto a esta investigación, también se revisó la obra de iniciación deportiva y su nivel de decepción en esta federación deportiva (Montoro Bombú, Ochoa Granda, et al., 2018)

Por otra parte, Sicilia Camacho, Á., \& Delgado Noguera (2002) citado en Lagla Chiluisa (2020) mencionan que "no se puede actuar de una manera fija ante los ambientes de aprendizaje y enseñanza que son abiertos. Los estilos de enseñanza más eficaces lo son en la medida en que con mayor frecuencia produce los efectos deseados, pero lógicamente es situacional" (p.27). a su vez para Sbert, (1969): "un maestro para enseñar a sus alumnos lo que desea que aprendan es preciso que él mismo haga ciertas cosas y que hay otras que son los aprendices quienes deben hacerlas.

No basta que estos vean cómo las hace el maestro, sino que éste debe formular cuidadosamente un plan de trabajo de las actividades propias del maestro y de aquellas que correspondan gradualmente a los aprendices" (p.22). importante es el criterio de Ortiz Ocaña, (2009) se refiere al "aprendizaje como un proceso de modificación relativamente permanente del modo de actuación del estudiante que modela y remodela su experiencia en función de la adaptación a los contextos en los que se concreta el ambiente con el que se relaciona, ya sea en la escuela o comunidad” (p.10).

Un dato que no podemos dejar de mencionar es el criterio de García Cortina, (2018) donde explica que "el aprendizaje es la facultad o habilidad que tienen los seres humanos para entender las cosas durante el proceso de adquisición de nuevos hábitos y comportamientos, se mejora significativamente cuando se aplica el modelo de Auto estructuración del conocimiento, cuyos atributos principales son: el ser objetivo, discreto, operante, optimista, el establecer la proporción y la concordancia” (p.6) 


\section{Técnica de carrera}

Vinuesa (1997) señala que "la carrera el deporte atlético clásico, puede considerarse, a la vez, cosa sencilla y difícil; sencilla porque se trata de una habilidad natural, que todo el mundo, incluso los menos dotados, lo realizan alguna vez en su vida" (p.14) (Lagla Chiluisa 2020)

Por lo que es necesario mencionar que técnica de carrera es la forma que tenemos de ejecutar los movimientos que intervienen en el gesto de la carrera, una buena técnica de carrera vendría a ser el modelo que un corredor puede realizar en el ciclo de su marcha desde el punto de vista biomecánico, motriz y energético. Una buena técnica de carrera no solo puede marcar la diferencia entre correr con o sin dolor, sino que también ayuda a mejorar marcas (Lagla Chiluisa 2020)

\section{Beneficios}

- Reduce el riesgo de sufrir cualquier tipo de lesión.

- Conseguir mayor velocidad y mejorar el rendimiento.

- Economizar al máximo la energía entrenando.

- Retrasar al máximo la aparición de la fatiga muscular.

- Aumentar la agilidad y la coordinación del cuerpo.

- Mejorar la eficacia de los movimientos.

- Adquirir una postura más cómoda y elegante al correr.

Por postura se entiende que son las variaciones de la colocación de unas partes del cuerpo con relación a otras, así como su capacidad para mantenerlas a pesar de las tensiones. Capacidad de mantener una colocación eficiente y fisiológica de las diferentes partes del cuerpo (Brigaud, 2015 citado en Lagla Chiluisa 2020). Una buena postura empieza desde la cabeza hasta los pies: mirada, cuello hombros, brazos, manos, espalda, cadera, piernas, rodillas y pies.

En la práctica es necesario tratar de aplicar dos aspectos como la demostración de la prueba en completo, y la explicación de los aspectos técnicos de forma simultánea, para luego demostrar el movimiento completo a diferentes velocidades y además el mismo entrenador va explicando los aspectos más importantes. Recuerde que en estas edades los niños necesitan más de la demostración que de la explicación (Lagla Chiluisa 2020).

Como es incuestionable, el atleta la va a realizar incorrectamente, con una técnica inadecuada. No importa, ese primer enfrentamiento con el movimiento completo es muy importante para él: le va a ofrecer su primera experiencia motora consiente de la técnica que va aprender y lo más importante, lo va a motivar al aprendizaje del movimiento fraccionado. En la actualidad este proceso es uno de los más referidos en las literaturas de la actividad física y el deporte(Raynier \& Lazaro de la Paz Arencibia, 2015 citado en Lagla Chiluisa 2020) y en el cual hay que seguir profundizando

\section{Metodologia.}

"El tema de investigación denominado: proceso enseñanza de la técnica de carrera en atletas categoría 10 a 11 años va encaminado a la formulación de nuevos procesos de entrenamiento mediante el apoyo de una guía de ejercicios, que fue eficiente en la etapa de enseñanza-aprendizaje de los atletas infantiles. Los elementos a investigar y para la formulación de las soluciones se utilizó un enfoque 
cuantitativo y cualitativo por el hecho del análisis de la realidad de los entrenadores y deportistas en cuanto a la enseñanza y aprendizaje, refiriéndonos al patrón cuantitativo esto permitió demostrar datos numéricos generados en tabulaciones de la encuesta, y la misma observación la cual cuenta con parámetros para evaluar la técnica de los atletas infantiles de la Federación Deportiva de Tungurahua. Además, se contó con el método científico para la obtención de un conocimiento teórico con validez y comprobación científica mediante el uso de instrumentos fiables; como, encuestas y la misma observación" (Lagla Chiluisa 2020).

\section{Muestra}

"La muestra de investigación estuvo centrada en la disciplina de atletismo y las edades comprendidas de 10 a 11 años. Constituida n-12 atletas de un total poblacional n-12 atletas que se mantienen permanentemente entrenando en las instalaciones de la Federación Deportiva de Tungurahua. Además, se contó con 3 entrenadores dando un total de 15 involucrados dentro de la F.D.T". (Lagla Chiluisa 2020).

\section{Procedimiento}

Para el correcto desarrollo de los procesos de enseñanza - aprendizaje basado en la técnica de carrera recurrimos a la observación con la ejecución de cuatro ejercicios básicos pero esenciales denominados SPRINT, para equiparar el progreso, se los plasmo en 5 semanas. En la Primera semana se cumplió con el ejercicio de carrera elevando muslos, el cual tuvo como objetivo instaurar al corredor el hábito de elevar rodillas. (Lagla Chiluisa 2020). La segunda semana se realizó el ejercicio de carrera elevando muslos con extensión de las piernas al frente el cual tuvo como objetivo aumentar la longitud de la zancada. En la tercera semana se ejecutó el ejercicio denominado carrera con extensión de las extremidades inferiores el objetivo que se buscó fue el rápido descenso de las extremidades inferiores, en la semana 4 se realizó el ejercicio de carrera de 50 que tuvo como objetivo la contribución el mejoramiento de la coordinación extremidades inferiores y superiores. (Lagla Chiluisa 2020).

Al término de la semana 5 se realizó la respectiva evaluación de la técnica de carrera con los parámetros establecidos. Cabe recalcar que en cada uno de los ejercicios se fueron observando parámetros fundamentales como posición de las extremidades superiores, posición del tronco, caderas, así como la mejora de la coordinación, concentración y relajación de los atletas, todos estos aspectos fueron planteados en la ficha de observación. (Lagla Chiluisa 2020).

El proceso metodológico se realizó con la respectiva observación que tuvo como objetivo la identificación de datos sobre los hechos en la técnica de carrera. Luego se procedió a la tabulación de resultados y la comprobación con ayuda de la prueba estadística de Shapiro-will para determinar los niveles de confiabilidad mediante T de Student, la aplicación de esta prueba se dio por el hecho de calcular muestras relacionadas y por tener una muestra pequeña menor a 30 individuos (Lagla Chiluisa 2020). Posteriormente se pasó a la elaboración de una guía de enseñanza de la técnica de carrera, que permitió un correcto desempeño de la técnica de las carreras planas. 
Tabla \# 1: Indicadores a evaluar por semanas de trabajo.

\section{FICHA DE EVALUACIÓN TÉCNICA}

Evaluación de cuatro ejercicios técnicos de carrera cada uno de ellos con 8 parámetros a evaluar con calificación de 1 al 4 siendo 4 la calificación más alta

Nombre:

Carrera con elevación de muslos

Parámetros

Distancia: $20-30 \mathrm{~m}$.

1. Movimiento fluido sin contracción excesiva de los planos musculares

2. Vista al frente

3. Ángulo de $90^{\circ}$ en el braceo y que no se pase de la línea central del cuerpo

4. Manos semicerradas

5. Tronco erguido

6. Caderas elevadas

7. La pierna elevada tiene que estar paralela al suelo con la rodilla flexionada a un ángulo de $90^{\circ}$, punta del pie mirando hacia el frente.

8. Pierna de apoyo totalmente extendida y apoyada sobre el metatarso.

Carrera elevando muslos con extensión de las piernas al frente

Distancia: 20 - $30 \mathrm{~m}$.

1. Movimiento fluido sin contracción excesiva de los planos musculares

2. Vista al frente

3. Ángulo de $90^{\circ}$ en el braceo y que no se pase de la línea central del cuerpo

4. Manos semicerradas

5. Tronco erguido

6. Caderas elevadas

7. La pierna elevada después recogida estará extendida y paralela al suelo con la punta del pie ligeramente inclinada hacia delante.

8. Pierna de apoyo extendida y apoyada sobre el metatarso.

Carrera con extensión de las extremidades inferiores

1. Movimiento fluido sin contracción excesiva de los planos musculares

2. Vista al frente

3. Ángulo de $90^{\circ}$ en el braceo y que no se pase de la línea central del cuerpo

4. Manos semicerradas

5. Tronco erguido

6. Caderas elevadas

7. La pierna elevada estará extendida a un ángulo de $45^{\circ}$ con respecto a la otra, la punta del pie ligeramente inclinada hacia delante.

8. Pierna de apoyo extendida y apoyada sobre el metatarso.

Carrera de $50 \mathrm{~m}$

1. Movimiento fluido sin contracción excesiva de los planos musculares

2. Vista al frente

3. Ángulo de $90^{\circ}$ en el braceo y que no se pase de la línea central del cuerpo

4. Manos semicerradas

5. Tronco erguido

6. Caderas elevadas

7. Coordinación entre extremidades superiores e inferiores.

8. Pierna de apoyo extendida y apoyada sobre el metatarso. 


\section{Resultados}

En función de ser objetivos en la presentación de los resultados, solo se mostrarán dos de los indicadores evaluados durante la observación del post y pre test, los cuales fueron tabulados tomando en cuenta los resultados obtenidos en cada uno de los parámetros evaluados. También fue importante precisar las alternativas de resultados estableciendo en cada una de ellas la frecuencia o repetición de la información. (Lagla Chiluisa 2020).

\section{Análisis de los resultados del pretest}

Gráfico \# 1: Resultados de pretest en la carrera con elevación de muslos.

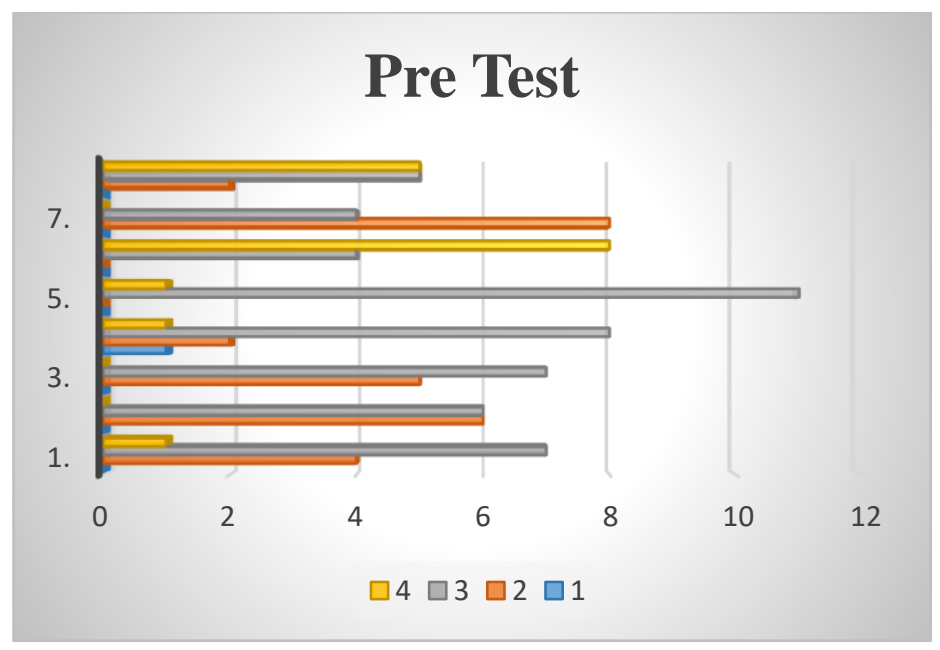

\section{Fuente: Elaboración propia}

El grafico muestra los resultados de las observaciones de la carrera con elevación de muslos en el pretest, existen 8 parámetros evaluados, del 1al 4 siendo 1 la calificación más baja y 4 la más alta calificación, por consiguiente en el parámetro 1, 4 atletas tienen la calificación de 2, 7 atletas con calificación de 3 y 1 solo atleta con calificación de 4 dándonos un total de 12 atletas evaluados. en el parámetro 2, 6 atletas tienen la calificación de 2, y 6 atletas con calificación de 3 dándonos un total de 12 atletas evaluados. En el parámetro 3, 5 atletas tienen la calificación de 2, y 7 atletas con calificación de 3 dándonos un total de 12 atletas evaluados. En el parámetro 4, un atleta tiene la calificación de 1, 2 atletas tienen la calificación de 2, 8 atletas con calificación de 3 y 1 solo atleta con calificación de 4 dándonos un total de 12 atletas evaluados. En el parámetro 5, 11 atletas tienen la calificación de 3 y 1 solo atleta con calificación de 4 dándonos un total de 12 atletas evaluados. En el parámetro 6, 4 atletas tienen la calificación de 3 y 8 atletas tienen la calificación de 4 dándonos un total de 12 atletas evaluados. En el parámetro 7, 8 atletas tienen la calificación de 2, 4 atletas tienen la calificación de 3 dándonos un total de 12 atletas evaluados. En el parámetro 8, 2 atletas tienen la calificación de 2, 5 atletas con calificación de 3 y 5 atletas con calificación de 4 dándonos un total de 12 atletas evaluados. (Lagla Chiluisa 2020). 
Gráfico \# 2: Carrera elevando muslos con extensión de piernas al frente Pre test

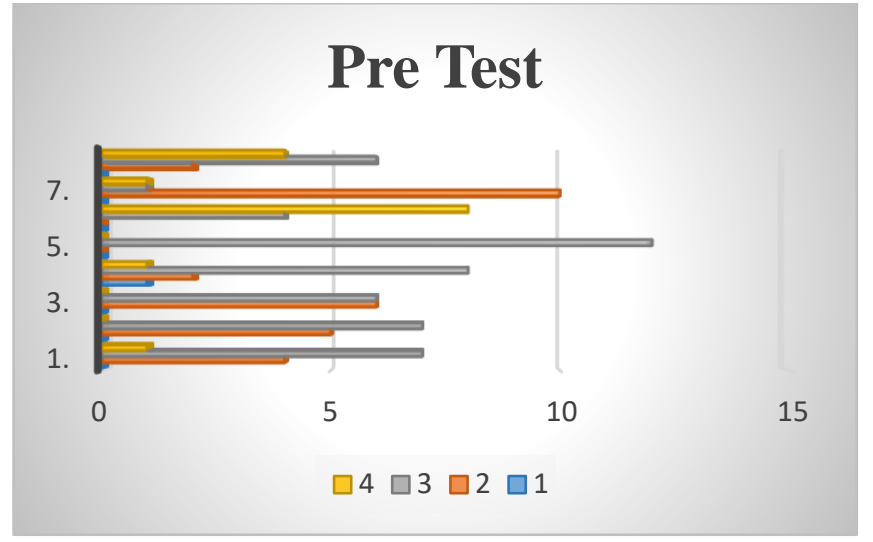

\section{Fuente: Elaboración propia}

Este gráfico muestra los resultados de las observaciones de la carrera elevando muslos con extensión de piernas al frente en el pretest, existen 8 parámetros evaluados, del 1al 4 siendo esta la más alta calificación, por consiguiente, en el parámetro 1, 4 atletas tienen la calificación de 2, 7 atletas con calificación de 3 y 1 solo atleta con calificación de 4 dándonos un total de 12 atletas evaluados. En el parámetro 2, 5 atletas tienen la calificación de 2, y 7 atletas con calificación de 3 dándonos un total de 12 atletas evaluados. En el parámetro 3, 6 atletas tienen la calificación de 2, y 6 atletas con calificación de 3 dándonos un total de 12 atletas evaluados. En el parámetro 4, un atleta tiene la calificación de 1,2 atletas tienen la calificación de 2, 8 atletas con calificación de 3 y 1 solo atleta con calificación de 4 dándonos un total de 12 atletas evaluados. En el parámetro 5, 12 atletas tienen la calificación de 3 dándonos un total de 12 atletas evaluados. En el parámetro 6, 4 atletas tienen la calificación de 3 y 8 atletas tienen la calificación de 4 dándonos un total de 12 atletas evaluados. (Lagla Chiluisa 2020).

\section{Análisis de los resultados del pos-test}

Gráfico\# 3: Carrera con elevación de muslos. Pos-test

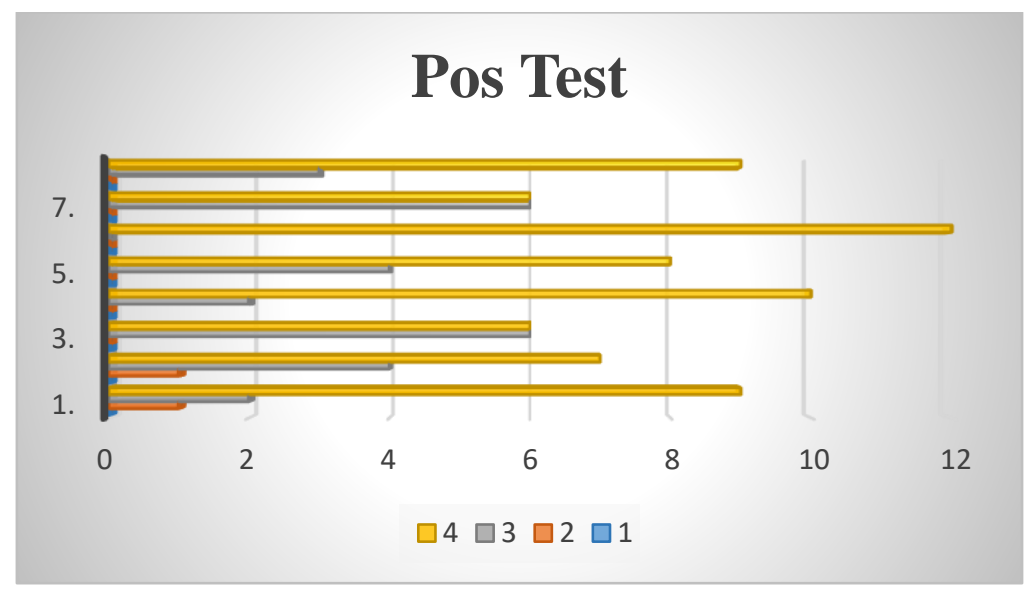

Fuente: Elaboración propia 
El gráfico muestra los resultados de las observaciones de la carrera con elevación de muslos en el pos test, son 8 parámetros evaluados, del 1al 4 siendo esta la más alta calificación, por consiguiente en el parámetro 1, un atleta tiene la calificación de 2, 2 atletas tienen la calificación de 3, y 9 atletas con calificación de 4 dándonos un total de 12 atletas evaluados. En el parámetro 2, un atleta tiene la calificación de 2, 4 atletas con calificación de 3 y 7 atletas la calificación de 4 dándonos un total de 12 atletas evaluados. En el parámetro 3, 6 atletas tienen la calificación de 3, y 6 atletas con calificación de 4 dándonos un total de 12 atletas evaluados. En el parámetro 4, 2 atletas tienen la calificación de 3, y 10 atletas tienen la calificación de 4, dándonos un total de 12 atletas evaluados. En el parámetro 5, 4 atletas tienen la calificación de 3 y 8 atletas tienen la calificación de 4 dándonos un total de 12 atletas evaluados. En el parámetro 6, 12 atletas tienen la calificación de 4 con un total de 12 atletas evaluados. En el parámetro 7, 6 atletas tienen la calificación de 3, y 6 atletas la calificación de 4 dándonos un total de 12 atletas evaluados. En el parámetro 8, 3 atletas tienen la calificación de 3, y 9 atletas tienen la calificación de 4 dándonos un total de 12 atletas evaluados. (Lagla Chiluisa 2020).

Gráfico \# 4: Carrera elevando muslos con extensión de piernas al frente. Pos-test

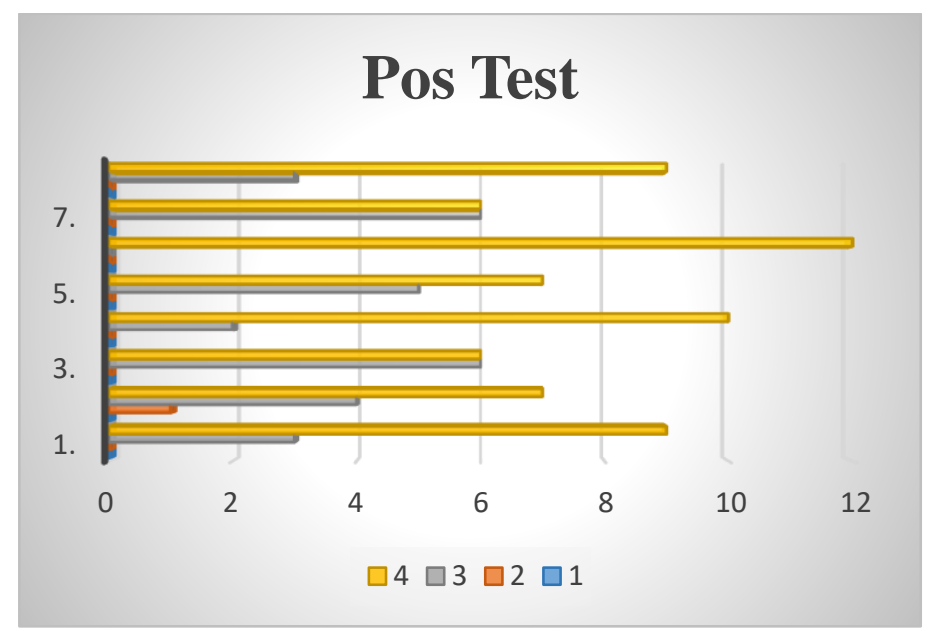

Fuente: Elaboración propia

La tabla 6: muestra los resultados de las observaciones de la Carrera elevando muslos con extensión de piernas al frente en el pos test, son 8 parámetros evaluados, del 1al 4 siendo esta la más alta calificación, por consiguiente, en el parámetro 1, 2 atletas tienen la calificación de 3, y 10 atletas con calificación de 4 dándonos un total de 12 atletas evaluados. En el parámetro 2, un atleta tiene la calificación de 2, 4 atletas con calificación de 3 y 7 atletas la calificación de 4 dándonos un total de 12 atletas evaluados. En el parámetro 3, 6 atletas tienen la calificación de 3, y 6 atletas con calificación de 4 dándonos un total de 12 atletas evaluados. En el parámetro 4, 2 atletas tienen la calificación de 3, y 10 atletas tienen la calificación de 4, dándonos un total de 12 atletas evaluados. En el parámetro 5, 5 atletas tienen la calificación de 3 y 7 atletas tienen la calificación de 4 dándonos un total de 12 atletas evaluados. En el parámetro 6, 12 atletas tienen la calificación de 4 con un total de 12 atletas evaluados. En el parámetro 7, 6 atletas tienen la calificación de 3, y 6 atletas la calificación de 4 
dándonos un total de 12 atletas evaluados. En el parámetro 8, 3 atletas tienen la calificación de 3, y 9 atletas tienen la calificación de 4 dándonos un total de 12 atletas evaluados. (Lagla Chiluisa 2020).

\section{Discusión}

Después de realizado el análisis de los resultados de cada una de las pruebas realizadas pudimos constatar que para el pre test la carrera elevando muslos, los resultados muestran que el desempeño técnico de la carrera elevando muslos, los atletas analizados presentaban errores técnicos muy básico como la visión al frente, déficit en la zona de apoyo con los metatarsos, en la mantención del ángulo de 90 grados en el braceo y un déficit en la posición de la cadera y tronco,(Montoro Bombú, Quizhpe Luzuriaga, et al., 2018 citado en Lagla Chiluisa 2020).) demostrando una falta de fuerza notada en esos planos musculares, por otra parte los resultados también mostraron que hay que realizar un énfasis marcado en el trabajo de los brazos, los cuales se cruzan de la línea central del cuerpo y no mantiene el ángulo de 90grados, luego de aplicado una guía de ejercicios se pudo mejorar los resultados de la segunda observación siendo validados los datos mediante la prueba estadística de Shapiro-Will para determinar los niveles de confiabilidad y mediante T de Student la cual comprobó que hay una diferencia significativa entre el pre test y el post test, por lo cual se concluye que el test del ejercicio carrera con elevación de muslos si tiene efectos significativos sobre la mejora de los atletas infantiles. De hecho, los atletas en promedio mejoraron de 22,8333 puntos a 29,5000 puntos. (Lagla Chiluisa 2020).

Para el caso de la carrera elevando muslos con extensión de las piernas, los resultados muestran que para el desempeño técnico, los atletas analizados presentaban ciertos errores técnicos básicos como son el ángulo de braceo que debe ser a 90 grados y no se pase de la línea central del cuerpo, déficit en la posición del tronco y en la posición de la cadera, y ciertos errores técnicos en zona de apoyo en los metatarsos demostrando una falta de fuerza notada en esos planos musculares luego de aplicado una guía de ejercicios se pudo mejorar los resultados de la segunda observación siendo validados los datos mediante la prueba estadística de Shapiro-Will para determinar los niveles de confiabilidad y mediante $\mathrm{T}$ de Student la cual comprobó que hay una diferencia significativa entre el pre test y el post test, por lo cual se concluye que el test del ejercicio carrera elevando muslos con extensión de piernas al frente si tiene efectos significativos sobre la mejora de los atletas infantiles de hecho los atletas en promedio mejoraron de 22,6667 puntos a 29,3333 puntos. (Lagla Chiluisa 2020).

Para el caso de la carrera con extensión de las extremidades inferiores los resultados muestran que para el desempeño técnico, los atletas analizados presentaban ciertos errores técnicos básicos como son el ángulo de braceo que debe ser a 90 grados y no se pase de la línea central del cuerpo, déficit en la apertura de las manos, posición del tronco y de cadera demostrando una falta de fuerza notada en esos planos musculares luego de aplicado una guía de ejercicios se pudo mejorar los resultados de la segunda observación siendo validados los datos mediante la prueba estadística de Shapiro-Will para determinar los niveles de confiabilidad y mediante T de Student la cual comprobó que hay una diferencia significativa entre el pre test y el post test, por lo cual se concluye que el test del ejercicio Carrera con extensión de las extremidades inferiores si tiene efectos significativos sobre la mejora de los atletas infantiles, de hecho los atletas en promedio mejoraron de 24,1667 puntos a 30,1667 puntos (Lagla Chiluisa 2020). Para el caso de la carrera de 50 m., los resultados muestran que para el 
desempeño técnico, los atletas analizados presentaban errores técnicos básicos como un déficit en la vista al frente, en la mantención del ángulo de 90 grados en el braceo y que no se pase de la línea central del cuerpo, déficit en la apertura de las manos, posición del tronco demostrando una falta de fuerza notada en esos planos musculares luego de aplicado una guía de ejercicios se pudo mejorar los resultados de la segunda observación siendo validados los datos mediante la prueba estadística de Shapiro-Will para determinar los niveles de confiabilidad y mediante T de Student la cual comprobó que hay una diferencia significativa entre el pre test y el post test, por lo cual se concluye que el test del ejercicio Carrera de $50 \mathrm{~m}$. si tiene efectos significativos sobre la mejora de los atletas infantiles, de hecho los atletas en promedio mejoraron de 25,5833 puntos a 30,5833 puntos. De esta manera podemos determinar que gracias a la aplicación de una guía de ejercicios predominan notables mejoras en el rendimiento técnico. Se produjo una recuperación aumentada de los depósitos energéticos de la musculatura y del organismo en general (Chango-Sigüenza \& Montoro-Bombú, 2018), lo que provocó que posteriormente mejorara significativamente la técnica de carrera en los atletas infantiles, esto se pudo comprobar mediante la aplicación de estadística en base a la prueba estadística de Shapiro - Will y T de Student.

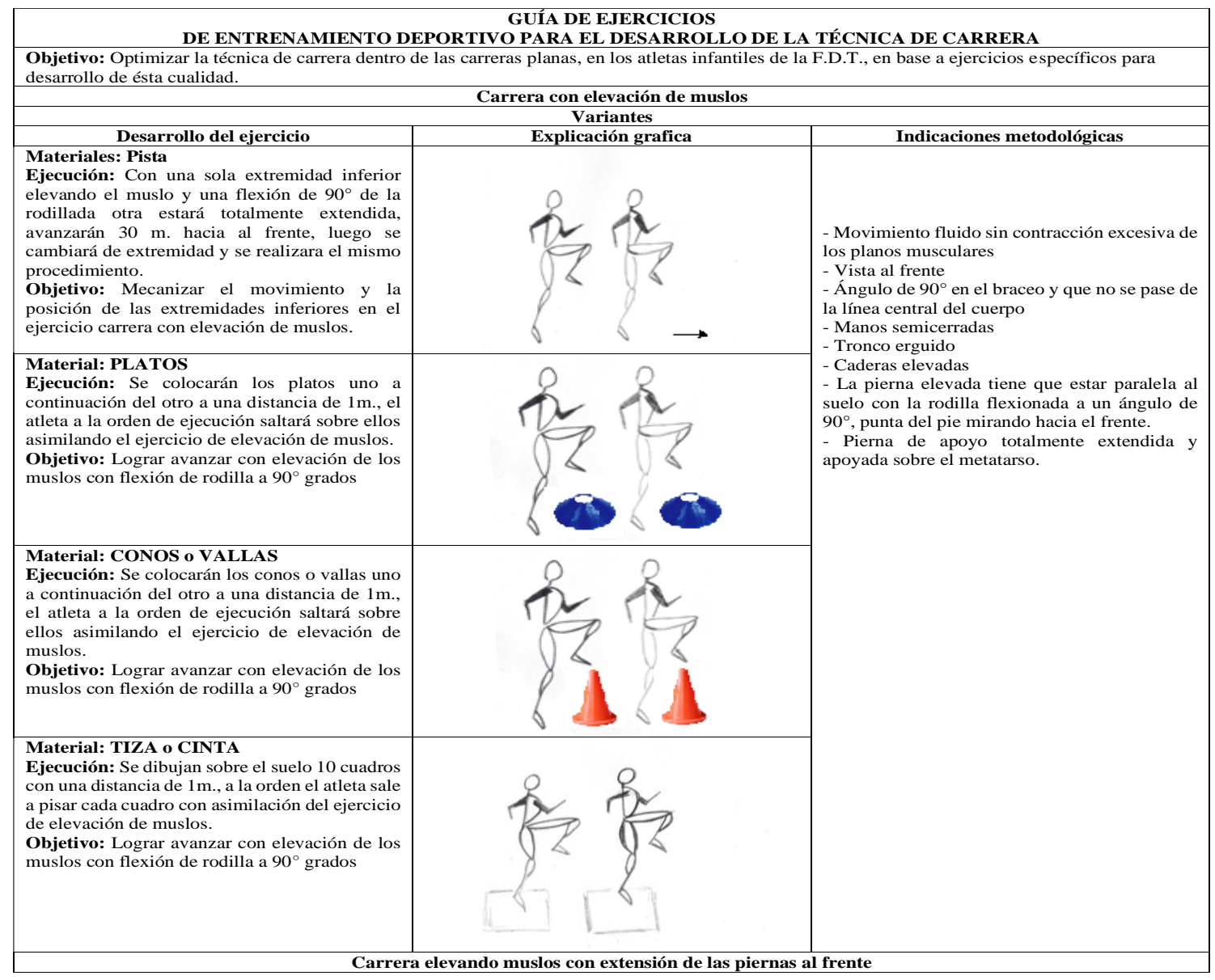




\section{Conclusiones}

Después de culminada la investigación, se llegó a las siguientes conclusiones:

- $\mathrm{Al}$ analizar referentes teóricos de acuerdo a los procesos de enseñanza - aprendizaje de la técnica de carrera en la disciplina de atletismo en el país nos dimos cuenta que, son bastante escasos, por ende, los entrenadores tienen insuficientes bases sobre como impartir una práctica adecuada en cuanto a la mejora de la técnica de carrera hacia los atletas infantiles.

- La guía de ejercicios, creada con ejercicios específicos en la técnica de carrera ayudaron al atleta al desarrollo de esta destreza.

- Las adecuaciones que se dieron a las prácticas para la mejora de la técnica de carrera fueron las esperadas, por el hecho de que se pudo observar una mejora en las 5 semanas.

\section{Referencias Bibliográficas}

Alex Mauricio Carrillo Aguagallo, Raynier Montoro Bombú, Pablo David Lincango Iza, Daniel Mon López, Edgardo Romero Frómeta, \& Marcos Elpidio Pérez Ruiz. (2018, septiembre). Efectos del método continuo-extensivo para potenciar la resistencia aeróbica en trail running y fondo. http://scielo.sld.cu/scielo.php?script=sci_arttext\&pid=S0864-03002018000300010

Cevallos Camacho, T. (2014). Programa de Iniciación deportiva en el atletismo para mejorar los procesos de Enseñanza - Aprendizaje en los alumnos de la Unidad Educativa Machachi, cantón Mejía, provincia de Pichincha durante el año lectivo 2014 - 2015. Latacunga.

Chango-Sigüenza, M., \& Montoro-Bombú, R. (2018). Artículo Original El desarrollo de las zonas funcionales en la marcha deportiva y las carreras de fondo Development of functional areas in middle-fund distance run and sport march (Vol. 14). http://accion.uccfd.cu

Inelvis Romero Pileta, Hirbins Manuel Dopico Pérez, Idalmis Fernández Téllez, Raynier Montoro Bombú, Enrique Chávez Cevallos, \& Wilson Teodoro Contreras Calle. (2019, junio). Análisis integral de la motivación en boxeadores. http://scielo.sld.cu/scielo.php?script=sci_arttext\&pid=S0864-03002019000200056

Lagla Chiluisa, M. D., \& Bombú Montoro, R. (2020). Estado actual del proceso enseñanzaaprendizaje de la técnica de carrera de los atletas de la categoría 10 a 11 años de la Federación Deportiva de Tungurahua. https://repositorio.uta.edu.ec/bitstream/123456789/31026/3/0503321887-Diego Mauricio Chiluisa Lagla .pdf

Montoro Bombú, R., Ochoa Granda, E., Quizhpe Luzuriaga, V., \& Valverde Jumbo, L. (2018). Valoración del rendimiento físico inicial y acciones estratégicas para disminuir la deserción deportiva en el atletismo femenino de la federación deportiva de Loja. Ciencia Digital, 2(4), 1-12. https://doi.org/10.33262/cienciadigital.v2i4.206

Montoro Bombú, R., Quizhpe Luzuriaga, V., Ochoa Granda, E., \& Medina Ramírez, E. (2018). Invariantes del contenido a tener en cuenta durante la enseñanza de los fundamentos técnicos de las carreras. Ciencia Digital, 2(4), 125-130. 
ISSN: 2600-5859

Vol. 3, $\mathrm{N}^{\circ} 1.2$, p. 123-136, marzo, 2020

https://doi.org/10.33262/cienciadigital.v2i4.214

Narváez Galván, E. F. (2013). La evaluación pedagógica y su incidencia en el desarrollo competitivo en las pruebas de medio fondo en los atletas pre-juveniles de la Federación Deportiva de Loja periodo 2013. Loja.

Raynier, M. B., \& Lazaro de la Paz Arencibia. (2015, febrero). Razonamientos sobre el rendimiento deportivo. Sus principales indicadores en corredores de 400 metros planos.

https://www.efdeportes.com/efd202/el-rendimiento-deportivo-en-corredores-de-400.htm

Sicilia Camacho, Á., \& Delgado Noguera, M. Á. (2002). Educación Física y Estilos de Enseñanza. En Análisis de la participación del alumnado desde un modelo socio-cultural del conocimiento escolar. INDE Publicaciones.

Tobar, M. A., \& Zurata, J. E. (2013). Guia metodológica para el perfeccionamiento de la técnica de la carrera en atletas fondistas del Club Trotahacheros del Municipio de Tuquerres-Nariño. Santiago de Cali. 
ISSN: 2600-5859

\section{PARA CITAR EL ARTÍCULO INDEXADO.}

Diego Mauricio, C. L., Edison Andrés, C. P., Fabricio Alexander, P. P., \& Janeth del Carmen, B. C. (2020). Proceso enseñanza de la técnica de carrera en atletas de la categoría 10 a 11 años. ConcienciaDigital, 3(1.2), 123-136. https://doi.org/10.33262/concienciadigital.v3i1.2.1191

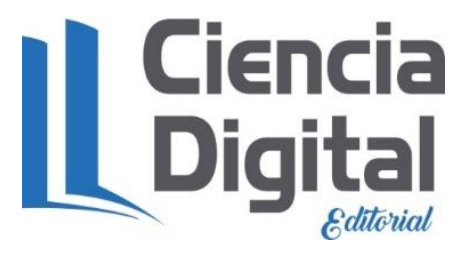

El artículo que se publica es de exclusiva responsabilidad de los autores y no necesariamente reflejan el pensamiento de la Revista Conciencia Digital.

El artículo queda en propiedad de la revista y, por tanto, su publicación parcial y/o total en otro medio tiene que ser autorizado por el director de la Revista Conciencia Digital.
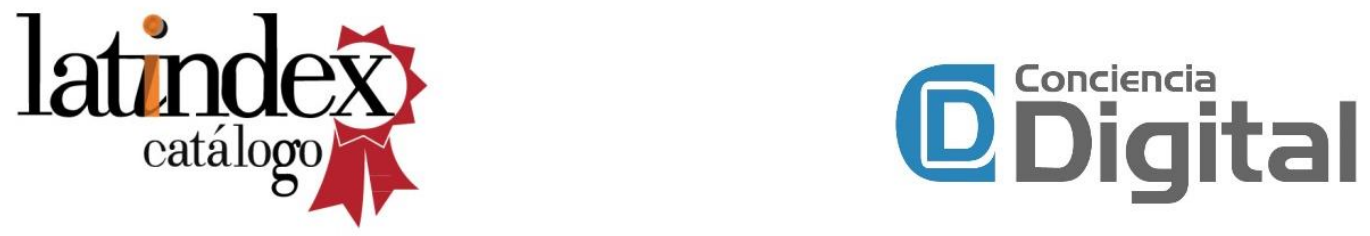